\title{
Heidi Armbruster, Keeping the Faith: Syriac Christian Diasporas
}

Canon Pyon, Sean Kingston Publishing, 2013, 279 p.

\section{Su Erol}

\section{OpenEdition}

\section{Journals}

Édition électronique

URL : http://journals.openedition.org/assr/26265

DOI : $10.4000 /$ assr.26265

ISSN : $1777-5825$

Éditeur

Éditions de l'EHESS

Édition imprimée

Date de publication : 31 décembre 2014

Pagination : 111

ISBN : 978-2-7132-2467-6

ISSN : 0335-5985

\section{Référence électronique}

Su Erol, "Heidi Armbruster, Keeping the Faith: Syriac Christian Diasporas », Archives de sciences sociales des religions [En ligne], 168 | 2014, mis en ligne le 30 mars 2015, consulté le 22 septembre 2020. URL : http://journals.openedition.org/assr/26265 ; DOI : https://doi.org/10.4000/assr.26265

Ce document a été généré automatiquement le 22 septembre 2020.

(c) Archives de sciences sociales des religions 


\section{Heidi Armbruster, Keeping the Faith: Syriac Christian Diasporas}

Canon Pyon, Sean Kingston Publishing, 2013, 279 p.

Su Erol

\section{RÉFÉRENCE}

Heidi Armbruster, Keeping the Faith: Syriac Christian Diasporas, Canon Pyon, Sean Kingston Publishing, 2013, 279 p. 
1 Les aspects sociologiques, politiques ainsi qu'anthropologiques de la communauté syriaque orthodoxe installée depuis plusieurs décennies en pays de diaspora n'ont guère été abordés par les chercheurs. Or, on pourrait ajouter plus généralement que les diasporas des chrétientés orientales n'ont pas non plus retenu l'attention de la recherche jusqu'à présent. L'ouvrage de l'anthropologue Heidi Armbruster cherche à combler cette lacune avec une recherche s'étalant sur une longue durée (l'auteur se sert des données ethnographiques qu'elle a recueillies depuis les années 1990 à aujourd'hui en les mettant en comparaison les unes avec les autres) basée sur trois terrains différents, à savoir Tur Abdin (foyer historique de la communauté), Berlin et Vienne.

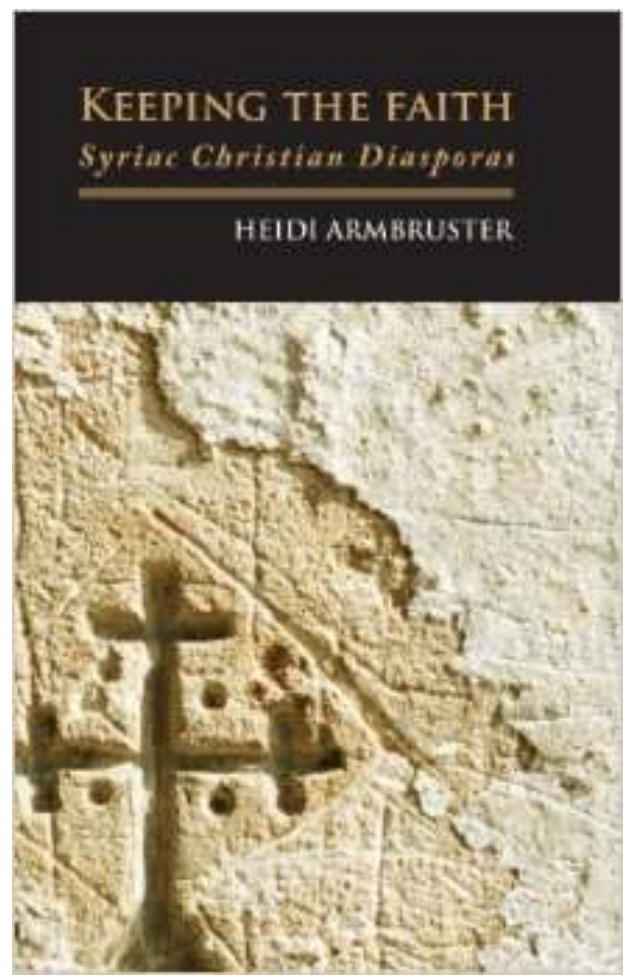

2 Précisons d'abord que ce travail adopte théoriquement «l'approche transnationale », qui propose de voir la migration comme une expérience subjective des individus concernés, pour mieux étudier leurs transformations identitaires. À la différence des théories à grande échelle, sa particularité vient du fait qu'elle privilégie les microcontextes, mettant au centre de son analyse les échanges de relations et de pratiques transfrontalières qui transcendent le cadre national. Elle insiste, dans ce cadre, sur la construction d'une nouvelle ethnographie qui se baserait plutôt sur les expériences vécues des sujets. En effet, l'ouvrage part de l'idée que l'expérience historique possède un caractère cumulatif plutôt que compartimenté et que les histoires de la prémigration peuvent avoir un effet important sur la construction identitaire des membres vivant actuellement dans le contexte postmigratoire.

3 Avant de présenter ses données, Armbruster fait un survol historique rapide de la communauté pour bien la situer dans sa temporalité. Le chapitre consacré à l'histoire est particulièrement bien construit car, à la différence d'autres travaux, une place considérable est accordée aux rapports entretenus avec les aghas kurdes, ces acteurs locaux dont l'influence est non négligeable dans la donne politique de la région de Tur Abdin. Sur ce point, je me permets d'ajouter aux données de l'auteur - datant de 2013 le résultat récent des élections municipales de Turquie, ayant lieu en mars 2014, qui a élu une maire syriaque à Mardin. Cet événement inattendu a rendu la visibilité politique des Syriaques de la région plus évidente que jamais.

4 Cela dit, revenons à la composition de l'ouvrage. La deuxième partie nous invite à reconsidérer l'identité du groupe dans son contexte local et transnational à travers trois éléments principaux marquant la particularité syriaque: l'histoire de la persécution liée à une certaine éthique du sacrifice, le maintien de la langue maternelle (syriaque) et celui de la croyance chrétienne. Selon l'auteur ayant séjourné pendant longtemps dans l'un des monastères de Tur Abdin, les nonnes du monastère 
s'identifient généralement aux saints et martyrs de la communauté quand elles parlent de leurs expériences de persécution datant de l'époque ottomane et se réfèrent fréquemment à l'hagiographie syriaque, en rétablissant ainsi leur supériorité morale à l'égard de celle des musulmans (p. 56). Dans ce cadre, les récits de seyfo (nom local des massacres de 1915 perpétrés sur les Syriaques) renforcent davantage les liens communautaires à travers le sentiment de victimisation collective. Le deuxième élément de la particularité communautaire, le maintien de la langue syriaque, symbole presque organique de l'extension du peuple, est directement associé à la reproduction de la famille ainsi qu'à la construction de l'ethnicité. L'enseignement de la langue maternelle est devenu une part importante de l'éducation monastique, destiné aujourd'hui aux jeunes venant des pays de diaspora pour des courtes durées, « contaminés " par les valeurs du monde occidental. Quant au troisième élément, défini selon le mot de l'auteur, «l'ethos de la croyance chrétienne », il se caractérise par une conduite morale qui doit contenir, en principe, des qualités diverses telles que la fermeté, la loyauté, le sacrifice, l'engagement et le dévouement, qualités qui déterminent également les limites de ce qui est du "suryoyotho" (les Syriaques de diaspora se définissent eux-mêmes par le terme syriaque de "Suryoyo". «Suryoyotho » veut dire alors le fait d'appartenir à la grande famille suryoyo). L'auteur met ici un accent spécial sur les questions du genre et constate qu'au niveau de l'organisation par sexe, existent des nettes distinctions, dont la plus concrète est celle de la division du travail au sein du monastère. Une forte domination masculine $y$ persiste, représentée notamment par le clergé qui prétend être le seul détenteur de la sagesse divine.

5 Dans la deuxième partie de son ouvrage, Armbruster se concentre sur le processus de l'émigration et y consacre un chapitre aux discussions relatives à la formulation d'une identité à caractère "ethnonational» abordant notamment les années après l'émigration en Allemagne et en Autriche (p.131-161). On assiste par exemple dans le contexte allemand à un véritable soutien venant des Églises catholique et protestante du pays, soit sous la forme de pressions internationales en faveur des chrétiens persécutés, soit sous la forme de travaux de documentation relatifs aux violations des droits de l'homme. Dans le cas autrichien, on voit également la présence d'une Église catholique qui soutient fortement leur reconnaissance publique dans la société d'accueil ainsi que leur intégration au pays (p. 120-121).

D'autre part, à travers des récits de vie recueillis, on comprend que l'identité chrétienne des Syriaques est mal saisie par les Allemands ou les Autrichiens, qui sont évidemment mal informés sur d'autres formes dechrétientés ou d'ethnicités qu'englobe la société turque. Les migrants syriaques se plaignent donc d'avoir été perçus comme des "Turcs" immigrés et luttent continuellement pour faire reconnaître leur particularité ethnicoreligieuse, c'est-à-dire leur principal signe de démarcation avec les musulmans turcs. Il serait juste de noter aussi que leur " projet » de construction identitaire basée sur une ethnicité précise - assyrienne ou araméenne résulte notamment de cette volonté de se différencier des Turcs et même des Kurdes, avec qui ils partagent le même territoire migratoire (p. 129-130). En ce qui concerne les discussions identitaires, l'auteur attire notre attention également sur la présence de deux types d'élites - religieuse et séculière - qui se concurrencent entre elles pour accéder aux capitaux économiques et symboliques de la communauté, en fonction bien évidemment des conditions du pays où ils se sont installés [sur ce sujet voir notamment la thèse de doctorat de Naures Atto, Hostages in the Homeland, Orphans in the Diaspora: 
Identity Discourses Among the Assyrian Syriac Elites in the European Dispora, Doctoral Thesis, Leiden University, 2011] (p. 144). Toutefois, ces rhétoriques variées produites sur la nature de l'identité «idéale » du groupe contribuent davantage à la division interne (p. 147-154) au sein de la communauté.

7 La troisième partie de l'ouvrage porte une attention particulière à la famille, en suggérant que celle-ci détermine les frontières intracommunautaires du groupe révélant beaucoup d'indices sur la place de l'individu dans son groupe d'origine, telles que sa réputation, sa position socio-économique ou ses choix politiques. La famille se trouve toujours au cœur de la survivance communautaire, dotée d'un rôle presque sacré quand il est question du maintien de l'identité. Les cérémonies somptueuses de noce et de baptême apparaissent alors comme des traits distinctifs qui séparent les membres de la communauté des Allemands ou des Européens tout en déterminant les statuts familiaux (p.163). Investir le capital économique est devenu ainsi la première occupation des membres en diaspora afin de garantir leur survivance dans le long terme et d'acquérir un grand prestige social pour mieux servir leur communauté (p.165). Bien que l'importance de la famille et son rôle unique dans le maintien des traditions aient été plusieurs fois soulignés par les religieux, l'auteur nous parle par ailleurs, d'une tension existante entre les conservateurs et les jeunes réformateurs qui défendent ardemment l'idée d'une modernisation de l'Église suivant une approche beaucoup plus laïque et modérée (p. 183).

8 Les récits de vie recueillis de différents membres d'une même famille (p.183-206) révèlent entre autres que les formes d'agency et de stratégies adoptées par les migrants pour s'intégrer à la société dominante présentent des caractéristiques différentes et parfois contradictoires. Adopter une forte éthique du travail pour mieux s'intégrer, respecter la hiérarchie familiale, purifier son espace privé de tout ce qui est «turc » à travers des pratiques quotidiennes (telles que parler le syriaque dans la vie courante, changer les noms turcs en syriaque), être conscient de son identité distincte dans un environnement multiculturel où "être différent " devient un atout sont d'ailleurs quelques exemples de ces stratégies. On constate qu'à Vienne, les jeunes hommes issus de la nouvelle génération se trouvent désormais au cœur de la mobilité sociale tout en restant fidèles à l'autorité familiale représentée par la figure du père (p. 214-215). Une histoire collective basée sur les privations, difficultés et épreuves vécues pendant les premières années de migration, marque presque toutes les narrations des interlocuteurs, comme un élément liant le passé et présent. Dans les récits de vie, autour desquels l'ouvrage est construit, l'identité est prise ainsi comme un ensemble de plusieurs niveaux à la fois collectif et personnel, loin d'être une entité fixe et immuable (p. 227).

9 L'ouvrage se termine par une évaluation générale de la réaction actuelle des Syriaques à la guerre civile éclatée en Syrie. Rappelant le fait que l'Assyrian Democratic Organization (ADO) a ouvertement critiqué le régime d'Assad (p. 230), l'auteur attire notre attention sur l'alliance traditionnelle toujours existante entre l'Église et le pouvoir officiel syrien. Enfin, considérant tous ces aspects politiques et sociologiques du phénomène migratoire ancrés dans la longue durée, Armbruster parle d'un "open-ended future » pour les Syriaques qui luttent notamment entre les nécessités du monde moderne et celles du monde communautaire caractérisé par l'extrême poids des traditions. 